\section{Controlling the flow of electromagnetic waves}

\author{
E.N.Economou
}

Foundation for Research and Technology - Hellas (FORTH) and Department of Physics, Univ. of Crete

We shall not cease from exploration and the end of our exploring will be to arrive where we started and know the place for the first time.

\section{T.S. Eliot}

\section{Waves in periodic media}

$T$ he importance of waves in the natural and the man-made $I$ world can hardly be overestimated. We see and we hear through waves. We transmit information through waves. We understand and control the electrical, magnetic and other properties of metals and semi-conductors through electronic waves. The latter propagate most often under the influence of a periodic (in space) potential due to the crystalline placement of the ions.

Waves in periodic media exhibit usually some common characteristics: The most important is the possibility of appearance of stop bands (also called gaps) separating pass bands (also called bands). This means that the frequency axis may be divided into alternating regions of gaps and bands: In the former, waves cannot propagate (cannot even exist in an infinite periodic medium), while in the latter they propagate freely due to constructive interference of the waves scattered by the periodic medium. The gaps appear as a result of destructive interference and/or the possible existence of non-accessible regions in space. Furthermore, a local deviation from the periodicity may trap the wave around it giving rise to an eigenfrequency in the gap. Attractive (i.e. negative) deviations tend to pull states out and below the bands, while repulsive ones (i.e. positive) tend to push states out and above the bands.

This picture allowed us to understand the distinction between conductors and insulators and formed the basis of the creation of solid state electronics.

The fact that electromagnetic (EM) and acoustic (AC) waves do not naturally propagate in periodic media and the focus on electronic waves in solids delayed until the late 80s the study of propagation of EM and AC waves in periodic media[1-4]. Actually this problem came to attention indirectly as a way of studying experimentally the localization problem in random media ${ }^{1}$ or creating more efficient lasers[2]. Thus, through the helical path shown in Fig. 1 we arrived at the so called photonic band gap materials, or more simply photonic crystals [5-7], i.e. artificial periodic structures (with unit cell size ranging from about one micron to a few centimeters) exhibiting gaps (or pseudogaps) in the photon density of states (DOS) due to strong scattering and destructive interference; we arrived, so to say, where we started in the late 20 s and early 30 s.

A photon propagating in a photonic (PC) crystal is slowed down as its frequency approaches a gap, and finally its group velocity vanishes at the edge of a band as shown in Fig.2a. In other words, near the edge of a band, the behavior of a photon changes from that of a massless relativistic particle to that of a massive non-relativistic particle capable of been trapped around a local defect (see ref. 5, p. 86), like an electron in a doped semiconductor.

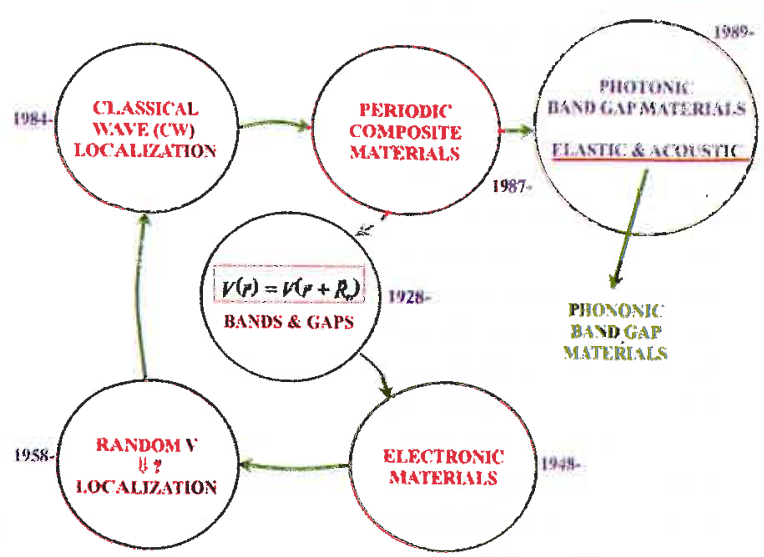

4. Fig. 1: The helical time path leading from Bloch's paper on electronic wave propagation in crystalline solids to photonic and phononic crystals.

Furthermore, no photon can exist inside a large (theoretically infinite) photonic crystal for frequencies belonging to a gap. As a result, an atom or a molecule implanted in the interior of a large photonic crystal would remain in the first excited state, since spontaneous emission is inhibited, provided that the relevant excitation energy (divided by Planck's constant) falls within the gap. The transmission coefficient of em waves of gap frequency exhibits a dramatic drop (see Fig.2b) providing thus a direct way to experimentally determine the gap.

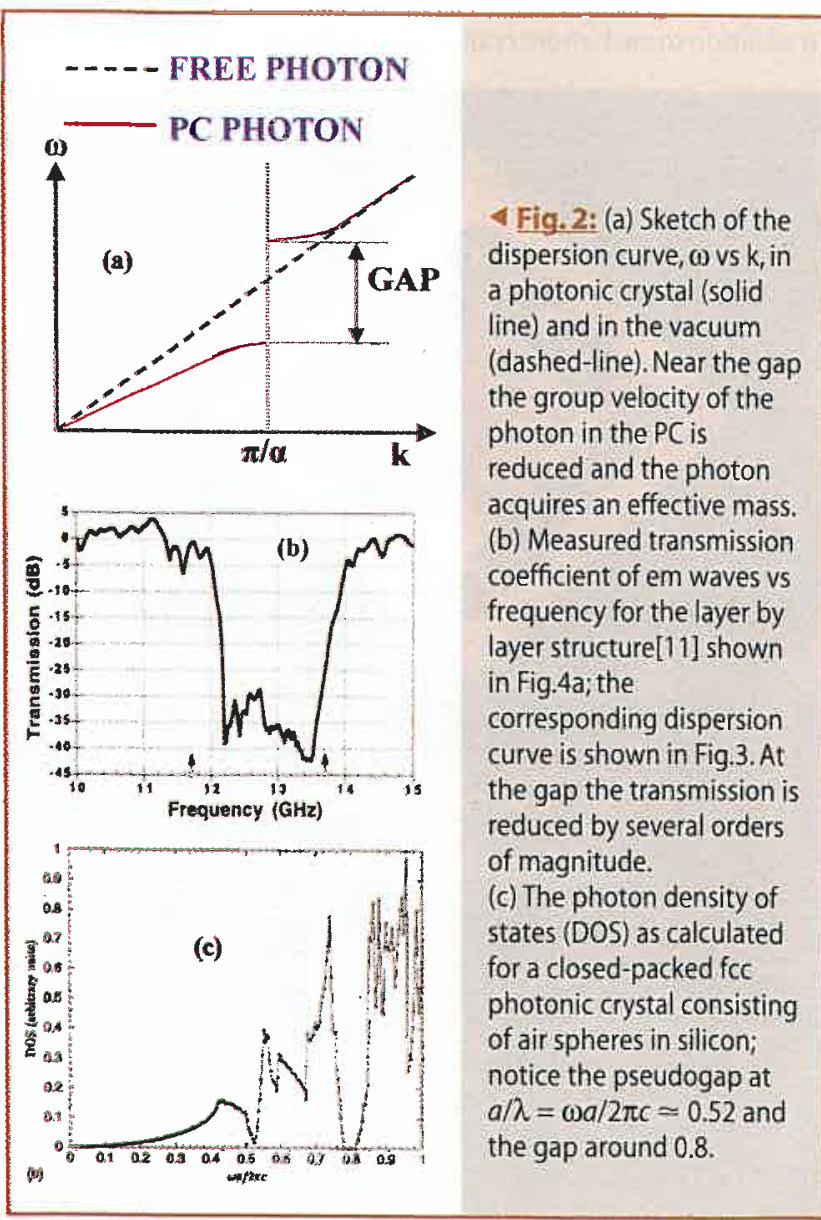


The photonic density of states (DOS) in a PC, in contrast to that of the free photon case, exhibits a complicated behavior with sharp peaks, abrupt dips, pseudogaps and true gaps as shown in Fig.2c. For low frequencies, i.e. wavelengths much larger than the lattice spacing, the PC appears to the wave as uniform and, consequently, the DOS is proportional to $\omega^{2}$ as in the free photon case. As the wavelength becomes comparable to the spatial periodicity, $a$, (and the sphere radius as well), the DOS exhibits strong variations. In particular, as shown in Fig. $2 c$, when the wavelength, $\lambda$, is equal to $1.92 a$ an almost gap (a pseudogap) is formed; finally, at $\lambda$ around $1.25 a$, a true narrow gap is created (gap over midgap approximately $5 \%$ ). This is a general feature of all known PCs: Gaps appear, if at all, when the photon wavelength is comparable to the spatial periodicity and the other characteristic lengths of the structure.

\section{Design and fabrication of photonic crystals}

In the 15 years since the initial idea $[1,2]$ of creating spectral em gaps in periodic media, photonic crystals have developed into an emerging technological field[7] offering the possibility of manipulating the emission, propagation, splitting, switching focusing, etc of em waves. In other words a novel tool has been added to facilitate perhaps our quest for the holy grail of all-photon telecommunications.

The creation of a spectral em gap requires the strong periodic spatial modulation of the dielectric function, $\epsilon$, and/or the magnetic permeability, $\mu$. This can be achieved by constructing composite materials consisting of two or more components with widely different $\epsilon$ and $\mu$. Usually, at high frequencies, $\mu$ is close to one for most (if not all) materials. Thus we are often left with the dielectric function $\epsilon$ as the only available material parameter. For the time being let us restrict ourselves to practically lossless dielectrics (although metals have also been used in the fabrication[6] of PCs). The simplest possible conceptual design of a photonic crystal is a composite consisting of spherical dielectric particles of high dielectric function $\left(\epsilon=\epsilon_{i}\right)$ placed periodically in a dielectric matrix [3] of low dielectric function $\left(\epsilon=\epsilon_{o}\right)$. The larger the ratio of dielectric functions, the stronger the scattering of em waves by the particles, and hence, the better the chances for

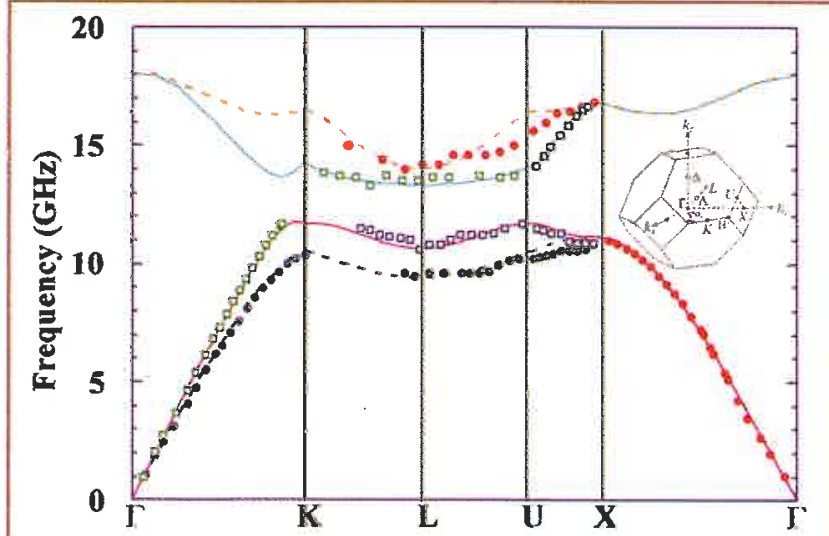

AF. 3: Frequency vs wavevector, as the latter changes along the straight line segments shown in insert, for em waves propagating in a PC of diamond-like symmetry (the layer by layer structure of Fig.4) with $\epsilon_{0}=1, \epsilon_{i}=9.6$ and volume fraction $f=0.26$. The various points are experimental data[11] and the various lines theoretical results based on plane wave expansions[11].

gaps creation. Strong scattering is also promoted when the so called Mie resonance conditions are satisfied, i.e. when the wavelength, $\lambda_{b}$ inside the particles is almost equal [3] to their diameter, d. In order for the strongly scattered waves to interfere destructively, the periodicity based Bragg condition must also be satisfied leading to the relation $\lambda \simeq 2 a$. These considerations justify our previous observation, that gaps appear, if at all, when the photon wavelength is comparable to both the period of the structure and the diameter of the scatterers. Thus to have gaps in the near infrared or at optical frequencies we need three-dimensional structures with spatial period in the micron or the submicron range respectively. Furthermore, the Mie and the Bragg conditions lead usually to volume fractions occupied by the high $\varepsilon$ material of around $20 \%$.

Several design parameters may influence the occurrence or not of gaps. We already mentioned the ratio $\epsilon_{i} / \epsilon_{o}$ (which must exceed $4.5)$ and the volume fraction, $f$, of the high $\epsilon$ material. Other parameters are: the lattice structure formed by the centers of the inclusions; the shape of the inclusions; the topology, i.e. whether each inclusion is fully surrounded by the host material (cermet topology) or whether the inclu-

In the 15 years since the initial idea... photonic crystals have developed into an emerging technological field. sions are connected with each other to form a continuous network (network topology).

Regarding the lattice structure one expects that the fcc symmetry would be the favorable one for gap formation. The argument goes as follows: In any arbitrary direction of propagation there are always gaps; if these gaps were independent of the direction (as in the case of the non-realizable spherical symmetry), we would have a full all-direction gaps; then fcc, being as close to spherical symmetry as it is possible for a periodic structure, must be the preferable symmetry. Detailed calculations and experiments with acoustic (scalar) and elastic (full vector) waves confirmed this prediction. However, em waves surprised the researchers: it turned out $[8,9]$ that diamond and diamond-like symmetry was the more favorable for em gap formation. There is no convincing explanation for this unexpected result, which may stem from the pure transverse nature of em waves and the consequent absence of a spherically symmetric scattered wave. Another odd behavior of em waves is related to the topology: In contrast to acoustic and elastic waves, the network topology, and not the cermet one, is the more favorable for em gap formation.

As the possible applications of photonic crystals grow, there is a proliferation of materials[6,7] and fabrication techniques[5$7,10]$ employed for their construction[6]: Thus semiconductors ( $\mathrm{Si}, \mathrm{Ge}, \mathrm{GaAs}, \mathrm{AlGaAs} / \mathrm{GaAs}$ ), oxides $\left(\mathrm{SiO}_{2}, \mathrm{TiO}\right)$ polymers (PMMA), protein crystals, metals, and other materials have been used as components or templates for PC fabrication. For the latter lithographic methods (using e-beams, focused ion beams, femtosecond laser pulses, $\mathrm{x}$-ray beams, holographic lithography) as well as self-assembled colloidal approaches combined with chemical etching have been employed. Extensive work has been devoted to the fabrication, study, and possible applications of twodimensional photonic crystals $[5,6]$ which allow easy design and construction of various devices, such as splitters, switches, etc. 
Fig. 4: Two examples of photonic erystals:

(a) The layer by layer design[1 1]; the structure repeats every four layers. (b) far right Inverted opals[10]; the space between self-organized silica particles is infiltrated by a high index dielectric and the silica particles are removed by chemical etching.
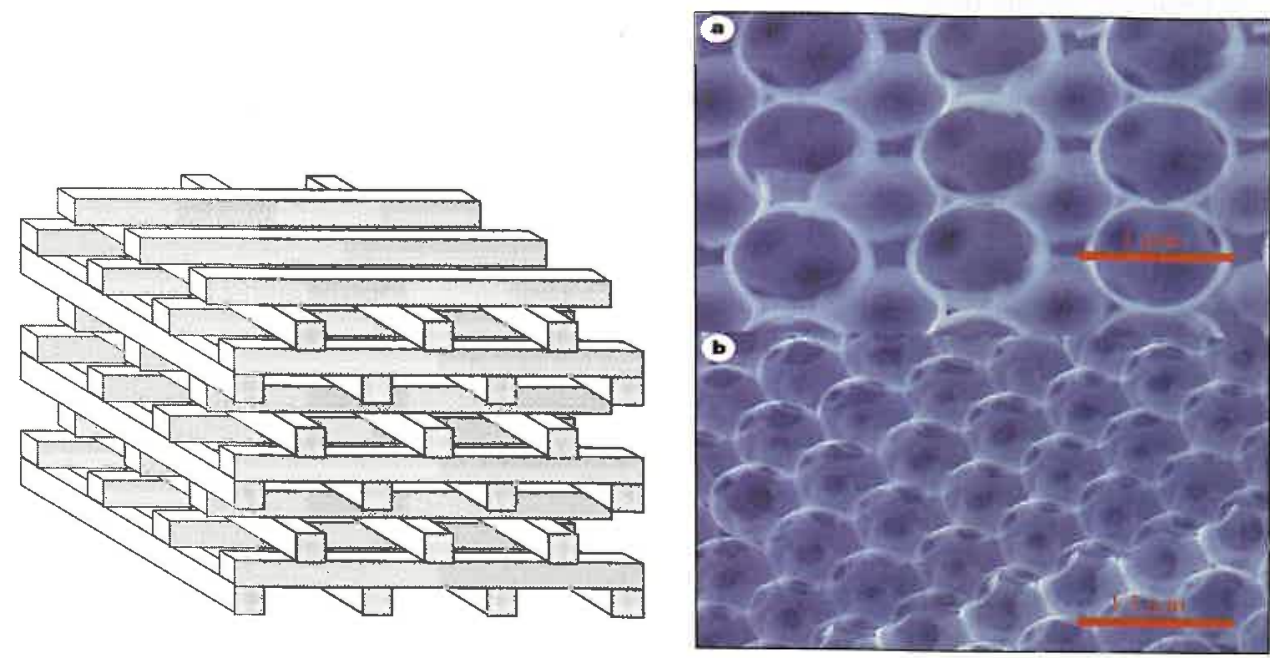

It must be pointed out that the design of PCs and PC based devices is greatly facilitated by numerical solutions of Maxwell's equations in these systems, based on various theoretical approaches such as plane wave expansions, transfer matrix techniques, multiple scattering methods, and finite difference time domain (FDTD) discretization. These computations involve neither uncontrollable approximations nor adjustable parameters; consequently they are very reliable, being in excellent agreement with experimental data as the example of Fig. 3 shows.

In Fig. 4 we show examples of two PCs which have pushed the midgap frequency to the near infrared or almost optical range by quite different fabrication techniques. The layer by layer structure[11] (Fig.4a) was recently minituarized[11] to micron length to produce a gap at telecommunication frequencies (i.e. wavelength of about $1.5 \mu$ ). The inverse opal structure[10,6] has naturally a lattice constant in the submicron range; however this important advantage makes the infiltration procedure by a high index dielectric difficult.

\section{Photonic crystals as negative (refractive) index materials (NIMs)}

Thirty five years ago, Veselago, a Russian physicist examined theoretically [12] what would happen to em waves propagating in a dispersive medium for which both $\mu(\omega)$ and $\epsilon(\omega)$ are negative and real. He found that the triad $\mathbf{k}, \mathrm{E}, \mathrm{H}$ is left-handed and not righthanded as in the usual case. As a result, the wavevector $k$ and the Poynting vector $\mathrm{S}=\mathrm{cE} \times \mathrm{H} / 4 \pi$ are opposite to each other, which means that energy and phase propagate in opposite directions. Consequently, the em wave refracted at the interface of an ordinary medium and a negative $\epsilon, \mu$ medium is in the "wrong" quarter plane as shown in Fig.5. This follows immediately by the conservation of energy and the parallel component of the wavevector and is equivalent to choosing the negative sign in the definition of the index of refraction as the square root of the product $\epsilon$ times $\mu$ (when both are negative). For this reason, materials with both $\epsilon$ and $\mu$ negative are referred to as negative index materials (NIMs) or left handed materials (LHMs), while ordinary dielectrics are called positive index materials (PIMs) or right handed materials (RHMs). The negative refraction permits the construction of flat lenses made from a slab of NIM, if such material can be found. Recently, Pendry[13] proposed a composite structure (consisting of metallic split rings which act as magnetic resonators and wires which act as electric resonators) for which effectively both $\epsilon$ and $\mu$ have negative values (over a lim- ited frequency range). Subsequently, Shelby et al.[14] constructed Pendry's structure and demonstrated experimentally negative refraction. Furthermore, Pendry (see p.329 in ref.6) argued that a slab of NIM can, under certain conditions, act as a superlens in the sense that it can focus more accurately than the wavelength limit (by "magnifying" the evanescent components which are usually lost).

Such a drastic extension of a field as established and "final" as electromagnetism has raised various objections which can be summarized as follows:

(a) Superlensing is not possible.

(b) Negative refraction seems to violate parallel momentum conservation, since momentum is parallel to $S$.

(c) Fermat's principle of minimum optical path is violated.

(d) Either negative refraction does not take place or causality and the speed of light is violated (because the outer ray of a beam of finite width has to move with infinite velocity in order to catch up with the inner ray in a negative refraction situation).

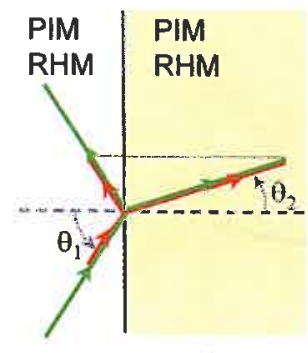

(1)
(2)

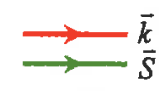

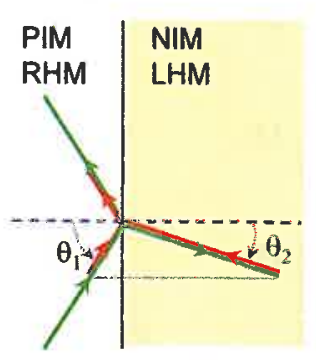

(1)

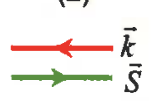

4Fig. 5: The Poynting vector $S$, showing the direction of energy flow, and the wave vector $k$, showing the direction of phase flow for a plane em wave at the interface of two different media. Energy of the refracted wave flows away from the interface, while the parallel components of $k$ of the incident, reflected, and refracted wave are equal. At the left is the case of two ordinary materials (with positive refractive index) while at the right is the case of positive/ negative index materials. 
(e) Absorption (which is always present, since Pendry's resonators are metallic) would invalidate Veselago's conclusions.

The jury's verdict regarding the first objection is not yet out; existing numerical results and analysis suggest that probably the superlensing, although real, would not be very significant.

The second objection is baseless, since in a dispersive medium the momentum is not [12] parallel to $S$; as a matter of fact in NIM the momentum is parallel to $\mathrm{k}$.

The third objection is also groundless since Fermat's principle refers to the extremum (and not necessarily to the minimum) of the optical path.

Finally the last two objections can be addressed more conveniently by invoking photonic crystals, since they are inherently lossless and-by proper choice of their structure and the frequency range - can exhibit negative refraction index behavior [15] in the following sense: The Poynting vector (averaged over the time period and the unit cell), which is parallel to the group velocity, can be antiparallel to the crystal wavevector $k$; under these circumstances the sign of the refraction index is negative.

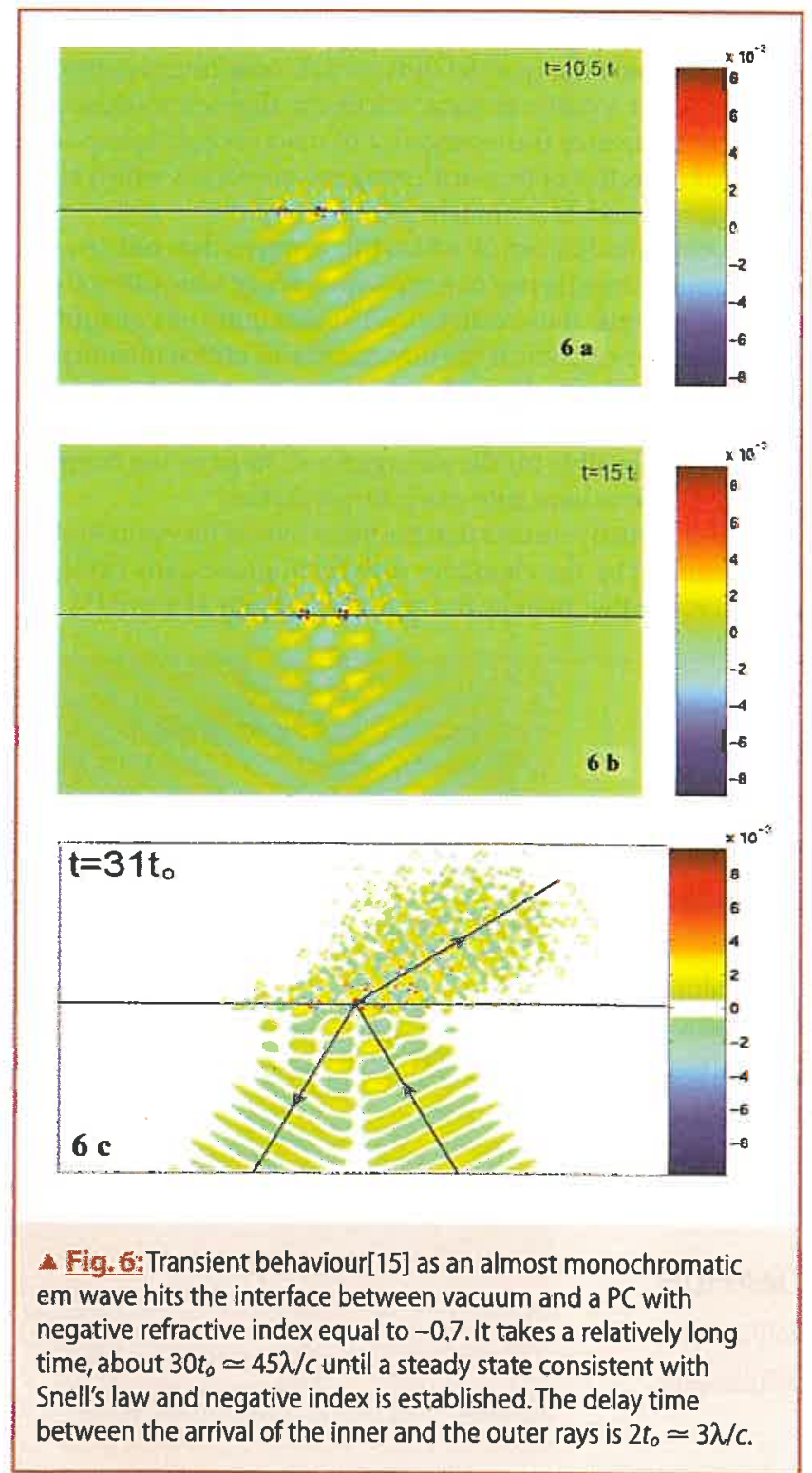

Recently Foteinopoulou et al. [15] considered a two-dimensional photonic crystal consisting of dielectric rods of radius $r=0.35 a$ and dielectric function $\epsilon=12.96$ placed in an hexagonal lattice of lattice constant $a$. In the range $a / \lambda$ between 0.57 and 0.61 the effective index of refraction is negative. A finite line source of em waves placed at an angle of 30 degrees relative to the interface between the vacuum and the $\mathrm{PC}$ starts emitting almost monochromatic radiation at $\lambda=1.73 a$ (corresponding to $\mathrm{PC}$ refractive index equal to -0.7), the inner ray of which reaches the surface of the PC after a time interval of $\Delta t=0.56 t_{0}$, while the outer one after $\Delta t^{\prime} \simeq 2.56 t_{o}$, where $t_{o} \simeq 1.5 \lambda / c$.

The results of numerically following the evolution of the em wave as a function of time is shown in Fig.6. The wave is trapped temporarily near the interface initially without indications of a specific direction of propagation (Fig.6a). Gradually signs of propagation along the direction determined by Snell's law (corresponding to the refractive index equal to -0.7) are shown (Fig.6b) and eventually after a long time (Fig. 6c) this direction is clearly followed. This numerical calculation not only demonstrated negative refraction; is has also shown that it takes a relatively long time for the wave to reorganize itself near the interface and to establish eventually a steady state consistent with Snell's law and negative refractive index. Thus the simplistic zero delay ray picture, on which the fourth objection was based, is not at all relevant to what really happens at a PIM/NIM interface as an em wave hits it.

In conclusion, the artificial periodic structures called photonic crystals have opened up new horizons in a subject as mature as the electromagnetic wave propagation in materials; in addition they constitute an emerging technological field which may play a significant role in future telecommunications.

This work was supported by EU grant DALHM.

\section{References}

[1] S. John, Phys. Rev, Lett., 58, 2486 (1987)

[2] E. Yablonovitch, Phys. Rev. Lett., 58, 2059 (1987)

[3] E. N. Economou and A. D. Zdetsis, Phys. Rev. B 40, 1334 (1989); M. Sigalas and E. N. Economou, J. of Sound and Vibration, 158, 377 (1992).

[4] E. Yablonovitch and T. J. Gmitter, Phys. Rev. Lett., 63, 1950 (1989)

[5] J. D. Joannopoulos et al., Photonic Crystals, Princeton Univ. Press, Princeton (1995)

[6] C. M. Soukoulis, ed., Photonic Crystals and Light Localization in the 21st Century, Kluwer, Dordrecht (2001)

[7] E. Yablonovitch, Scientific American, Dec.2001, p. 35

[8] K. M. Ho, C. T. Chan, and C. M. Soukoulis, Phys. Rev. Lett. 65, 3152 (1990)

[9] E. Yablonovitch, T. J. Gmitter, and K. M. Leung, Phys. Rev. Lett. 67, 2295 (1991)

[10] A. Blanco et al. Nature 405, 437 (2000)

[11] K. M. Ho et al, Sol. St. Comm. 89, 413 (1994); E. Ózbay et al, Phys. Rev. B50, 1945 (1994); S. Y. Lin et al., Nature 394, 251(1998); see also p. 83 in ref. 9

[12] V. G. Veselago, Sov. Phys. Usp. 10, 509 (1968)

[13] J. Pendry, Phys. Rev. Lett., 85, 3966 (2000) and in ref.9, p.329

[14] R. A. Shelby, D. R. Smith, S. Schultz, Science, 292, 77 (2001)

[15] P. Foteinopoulou et al., submitted to Phys. Rev. Lett., condmat/0210346. 